21st Particles and Nuclei International Conference (PANIC 2017)

International Journal of Modern Physics: Conference Series

Vol. 46 (2018) 1860033 (6 pages)

(C) The Author(s)

DOI: $10.1142 / \mathrm{S} 2010194518600339$

\title{
Shedding Light on Hexaquarks
}

\author{
M. Bashkanov* \\ School of Physics and Astronomy, University of Edinburgh, \\ James Clerk Maxwell Building, Peter Guthrie Tait Road, Edinburgh EH9 3FD,UK \\ mikhail.bashkanov@ed.ac.uk \\ T. Skorodko \\ Physikalisches Institut, University of Tuebingen, \\ Auf der Morgenstelle 14, D-72076 Tuebingen, Germany \\ H. Clement \\ Physikalisches Institut, University of Tuebingen, \\ Auf der Morgenstelle 14, D-72076 Tuebingen, Germany \\ D. P. Watts \\ School of Physics and Astronomy, University of Edinburgh, Address \\ James Clerk Maxwell Building, Peter Guthrie Tait Road, Edinburgh EH9 3FD,UK
}

Published 3 May 2018

\begin{abstract}
Several new findings in the four, five and six quark systems reheat the interest in the field of multiquark states (beyond the trivial $q \bar{q}$ and $q q q$ ). A lot of progress has recently been made in the $6 \mathrm{q}$ sector, on both the theoretical and experimental side. A resonance like structure observed in double-pionic fusion to the deuteron, at $\mathrm{M}=2.38 \mathrm{GeV}$ with $\Gamma=70 \mathrm{MeV}$ and $I\left(J^{P}\right)=0\left(3^{+}\right)$has been consistently observed in a wealth of reaction channels, supporting the existence of a resonant dibaryon state - the $d^{*}(2380)$. These studies include measurement of all the principle strong decay channels in pn collisions in the quasifree mode by the WASA-at-COSY and HADES collaborations. The internal structure of the $d^{*}(2380)$ is largely unknown. It can contain various "hidden color" $6 \mathrm{q}$ configurations, $\Delta \Delta$ molecular states with angular momentum $\mathrm{L}=0,2,4,6$ as well as meson-assisted dressed dibaryon structures. The large set of experimental data obtained to date gives some constraints on the internal structure of the $d^{*}(2380)$ dibaryon, but does not settle the issue. The $d^{*}$ is the only multiquark state which can be produced copiously at current facilities, offering unique access to information beyond its basic quantum numbers, particularly its physical size and internal structure.
\end{abstract}

Keywords: $d^{*}(2380)$; dibaryons.

\section{*Speaker}

This is an Open Access article published by World Scientific Publishing Company. It is distributed under the terms of the Creative Commons Attribution 4.0 (CC-BY) License. Further distribution of this work is permitted, provided the original work is properly cited. 


\section{Bashkanov et al.}

\section{Introduction}

The matter in the visible universe is comprised mainly of protons and neutrons, bound systems each containing 3 quarks. For almost half a century the only bound system containing 6-quarks established in nature was the deuteron, a molecule composed of a proton and a neutron.

The question whether there are more eigenstates in the system of two baryons than just the deuteron ground state has been around since the discovery of the deuteron. Seraches for dibaryon states have a considerable history (for a recent review see $\operatorname{Ref}^{1}$ ).

The recent exclusive and kinematically complete measurements performed by the Wasa-at-COSY collaboration revealed the first non-trivial 6-quark state - the $d^{*}(2380)$ hexaquark. This new exotic particle with quantum number $I\left(J^{P}\right)=0\left(3^{+}\right)$ was successfully observed in five two-pion production channels: $p n \rightarrow d \pi^{0} \pi^{0}, p n \rightarrow$ $d \pi^{+} \pi^{-}, p n \rightarrow p p \pi^{0} \pi^{-}, p n \rightarrow p n \pi^{0} \pi^{0}, p n \rightarrow p n \pi^{+} \pi^{-2,3,4,5,6}$ and in $p n$-elastic scattering reaction ${ }^{7,8}$. For the latter reaction a partial wave analysis was performed by the SAID group of George Washington University 7,8 .

Theoretical predictions of dibaryons, such as the $d^{*}$, have a long history. Calculations started with the pioneering work of Dyson and Xuong ${ }^{9}$ in 1964 and have developed in many studies since then $10,11,12,13,14,15,16,17,18,19,20,21,22,23,24,25$. Various configurations were suggested to describe the $d^{*}$ internal structure. Some can be tested experimentally.

\section{Structure of the $d^{*}(2380)$}

The data on the $d^{*}(2380)$ suggest that hadronically this object constitutes a $\Delta \Delta$ system bound by 80 - $90 \mathrm{MeV}$. Considering the dependence of the size from the binding energy $E_{B}$ and the mass of molecular object constituents, size $\sim 1 / \sqrt{m E_{B}}$, we should expect the $d^{*}(2380)$ to be 7 times smaller than the deuteron, $R_{\Delta \Delta} \sim 0.3 \mathrm{fm}$. Since overlapping $\Delta \Delta$ configurations cannot be differentiated from a genuine hexaquark ${ }^{27}$, one can treat the $d^{*}(2380)$ as a compact hexaquark state with somewhat extended tails from the $S-$ wave and $D-$ wave $\Delta \Delta$ configurations. At least $5 \% D$-wave $(L=2) \Delta \Delta$ branch contribution was evaluated by analysis of the $p n \rightarrow d^{*} \rightarrow d \pi^{0} \pi^{0}$ reaction in Ref. ${ }^{30}$ - a remarkable feature for the $80 \mathrm{MeV}$ subthreshold system. Detailed quark model calculations ${ }^{24}$ predict the $d^{*}(2380)$ to have a radius of $0.8 \mathrm{fm}$, indicating a compact hexaquark system. Conversely, Faddeev calculations based on purely hadronic interactions predict this state to have asymptotically a $D_{12} \pi$ configuration (rather than $\Delta \Delta$ ) representing a dilute molecular-like object $11,12,13$. The level ordering in these two schemes are also different. In a hexaquark picture the lowest lying $6 \mathrm{q}$ configuration is the most compact one, with the $S$ - wave $\Delta \Delta$ heavier and more extended and a $D-$ wave $\Delta \Delta$ heavier and more extended still. In the Faddeev picture the lightest configuration, the $D_{12} \pi$ is barely bound/unbound relative to the $N \Delta \pi$ threshold (2310 MeV - $70 \mathrm{MeV}$ below the $d^{*}$ mass $)$ having thus a very large size $\left(R_{N \Delta \pi} \sim 2.5 \mathrm{fm}\right)$. Therefore for this 
approaches the $\Delta \Delta$ configuration does not need to be as bound and compact as in the hexaquark picture and the heavy configuration $(\Delta \Delta)$ is the most compact one.

In vacuum the two theoretical approaches can hardly be distinguished, but they have opposite effects in nuclear matter. Due to Debye screening, the extended component of the $d^{*}$ will melt down in the dense nuclear medium. In the hexaquark picture the extended radial components will be the $D-$ wave $\Delta \Delta$ while in the Faddeev it is the $D_{12} \pi$ component. A lightening of the $d^{*}$ in nuclear matter would be expected for the first case and an increase in mass for the second.

Although the isospin relations for the decays into the various $N N \pi \pi$ channels are identical for intermediate $\Delta \Delta$ and $D_{12} \pi$ configurations, the decay into the isoscalar $[N N \pi]_{I=0}$ channel is expected to show strong differences. Whereas a $\Delta \Delta$ configuration has no decay to $[N N \pi]_{I=0}$ in leading order, the $D_{12} \pi$ configuration gives a $18 \%$ branching based on the corresponding branching of the decay $D_{12} \rightarrow N N$. Our measurement of the $N N \rightarrow[N N \pi]_{I=0}$ reaction gives no indication for a decay branch $d^{*}(2380) \rightarrow[N N \pi]_{I=0}$ yielding an upper limit of less than $9 \%{ }^{26}$. If it exists, a possible $D_{12} \pi$ configuration should predominantly decay into the $N N \pi$ channel with two nucleons in a relative $D-$ wave and a pion in relative $P-$ wave. Future partial wave analysis of the isoscalar single pion cross-section should allow further restriction of the $D_{12} \pi$ configuration. Ultimately, a measurement of the $d^{*}(2380)$ transition form-factor would enable the different possibilities for $d^{*}$ structure to be evaluated, including various exotic options such as a dominant di-quark or benzenelike hexa-quark structures ${ }^{27}$.

\section{3. $d^{*}$ photoproduction}

Before any attempt to measure the $d^{*}$ form-factor can be made it is important to determine the nature of its electromagnetic coupling. The simplest first step to achieve this is the photoproduction of the $d^{*}: \gamma d \rightarrow d^{*}$. The reaction $\gamma d \rightarrow d \pi^{0} \pi^{0}$ appears to be attractive, since conventional nucleon resonant processes are expected to be particularly small ${ }^{31,32}$, of the order of only $10 \mathrm{nb}$ at $T_{\gamma}=550 \mathrm{MeV}$ with a smooth energy dependence. The next "best" two-pion production channel $\gamma d \rightarrow d \pi^{+} \pi^{-}$has a background two orders of magnitude higher, peaking around the position of the $d^{*}$ due to the Kroll-Ruderman term, which is unfortunate for the $d^{*}$ photoproduction studies. The very first results on $\gamma d \rightarrow d^{*} \rightarrow d \pi^{0} \pi^{0}$ have recently appeared from Tohoku ${ }^{33}$ and Mainz ${ }^{34}$. They estimate the cross section of the $\gamma d \rightarrow d^{*} \rightarrow d \pi^{0} \pi^{0}$ reaction in the order of $20 \mathrm{nb}$ indicating $\Gamma\left(\gamma d \rightarrow d^{*}\right) \approx 0.6 \mathrm{keV}$. Both measurements currently experience large systematical uncertainties due to difficulties in removing background processes. Further measurements including different final states are crucial to firmly establish the photocoupling. The $d^{*}(2380)$ is a unique particle in many respects, one of which is a non-trivial photon coupling. Due to the small size of the fine-structure constant $\alpha \sim 1 / 137$ each extra photon contribution to a decay channel would normally be expected to result in around two orders of magnitude suppression in its probability. However, for the $d^{*}$ 
the case is the opposite. This is because the decay width $\Gamma\left(d^{*} \rightarrow \Delta \Delta\right) \sim 60 \mathrm{MeV}$ is large. Therefore the probability of each $\Delta$ to decay into $\Delta \rightarrow N \gamma$ is $\sim 0.6 \%$ considering the (high) probability to form a deuteron from the two nucleons from the $\Delta$ decay then a $d^{*} \rightarrow d \gamma \gamma$ width of order $\Gamma\left(d^{*} \rightarrow d \gamma \gamma\right) \sim 1 \mathrm{keV}$ is expected larger than $\Gamma\left(d^{*} \rightarrow d \gamma\right) \sim 0.6 \mathrm{keV}$. Similar strengths of the one- and two-photon electro-excitation cross-sections for the $d^{*}$ lead to interesting interference effects, which may be visible in the angular distributions of the decay products. Interference effects in $d^{*}$ electroproduction have the exciting potential to achieve sensitivity to the internal structure of the $d^{*}$.

\section{4. $d^{*}(2380)$ in the Nuclear medium}

The $d^{*}(2380)$ is robust enough to survive even in the nuclear environment $35,36,37,38$. It was recently demonstrated that $d^{*}$ can potentially form dibaryonic matter deep inside neutron stars(NS), participate in neutron star cooling and influence neutron star mergers ${ }^{39}$. The nuclear Equation of State (EoS) with explicit $d^{*}$ degrees of freedom is one of the few EoS on the market which fits the new limits imposed by the GW170817 ${ }^{40}$ gravitational wave NS merger measurement. Further studies of the $d^{*}$ behaviour in nuclear matter may be important to clarify it's role in NS merger dynamics. Such studies can be performed with $d^{*}$ photoproduction in nuclei. The main effect which limits the $d^{*}$ photoproduction cross section is a tiny overlap between two nucleons inside deuteron. When one probes the light-front wavefunctions of the deuteron where all of the six quarks have small relative separation, as in the deuteron form factor at high momentum transfer or in photodisintegration $\gamma d \rightarrow n p$ at high transverse momentum, the five "hidden-color" configurations of the deuteron mix due to gluon exchange and become equal in magnitude at asymptotic $Q^{2} \rightarrow \infty{ }^{41}$. For example, the observed $Q^{10} F_{d}\left(Q^{2}\right)$ scaling ${ }^{42}$ of the deuteron $\sqrt{A}\left(Q^{2}\right)$ form factor at high $Q^{2} 43$ is dominated by hidden-color configurations. This result can be derived by applying ERBL evolution ${ }^{44,45}$ to the five-component deuteron distribution amplitude $\phi_{d}\left(x_{i}, Q\right)$. At $Q^{2} \rightarrow 0$ the $\mid 6 q>$ configuration of the deuteron is only $\sim 0.15 \%{ }^{46}$ leading to a 3 orders of magnitude suppression in the $\gamma d \rightarrow d^{*}$ reaction cross section. This restriction can be overcome if we substitute the deuteron with a compact nucleus. Due to the large pn-pairing inside nuclei, the study of the $d^{*}$ medium modifications with photo-induced reactions in nuclei is expected to be pretty straightforward, provided one could find a reliable way to suppress backgrounds from quasi-free multi-pion production.

\section{Summary and outlook}

After a vast number of unsuccessful searches a non-trivial dibaryon resonance has now been found and its major decay channels identified. What is missing, is a measurement of its electromagnetic form factor, in order to learn about the size of this object - whether it is of molecular type or a compact six-quark entity. Further experiments at MAINZ and JLab are expected to resolve this question. 


\section{Acknowledgement}

We acknowledge valuable discussions with A. Gal, Yubing Dong and F. Huang on this issue. This work has been supported by STFC (ST/L00478X/1) and DFG (CL $214 / 3-1)$.

\section{References}

1. H. Clement, Prog. Part. Nucl. Phys. 93, 195 (2017).

2. M. Bashkanov et al., Phys. Rev. Lett. 102, 052301 (2009).

3. P. Adlarson et al., Phys. Rev. Lett. 106, 242302 (2011).

4. P. Adlarson et al., Phys. Lett. B 721, 229

5. P. Adlarson et al., Phys. Rev. C 88, 055208 (2013).

6. P. Adlarson et al., Phys. Lett. B 743, 325 (2015).

7. P. Adlarson et al., Phys. Rev. Lett. 112, 202301 (2014).

8. P. Adlarson et al., Phys. Rev. C 90, 035204 (2014).

9. F. J. Dyson and N.-H. Xuong, Phys. Rev. Lett. 13, 815 (1964).

10. M. Bashkanov, S. Brodsky and H. Clement, Phys. Lett. B 727, 438 (2013).

11. A. Gal and H. Garcilazo, Phys. Rev. Lett. 111, 172301 (2013).

12. A. Gal and H. Garcilazo, Nucl. Phys. A 928, 73 (2014).

13. A. Gal, Phys.Lett. B 769, 436 (2017).

14. H. Huang, J. Ping and F. Wang, Phys. Rev. C 89, 034001 (2014).

15. X. Q. Yuan, Z. Y. Zhang, Y. W. Yu and P. N. Shen, Phys. Rev. C. 60, 045203 (1999).

16. Woosung Park, Aaron Park, Su Houng Lee, Phys.Rev. D 92, 014037 (2015)

17. Hua-Xing Chen, Er-Liang Cui, Wei Chen, T.G. Steele, Shi-Lin Zhu, Phys. Rev. C 91, $025204(2015)$

18. Hongxia Huang, Pu Xu, Jialun Ping, Fan Wang, Phys. Rev. C 84, 064001 (2011)

19. Mei Chen, Hongxia Huang, Jialun Ping, Fan Wang, Phys. Rev. C 83, 015202 (2011)

20. Qi-Fang L, Fei Huang, Yu-Bing Dong, Peng-Nian Shen, Zong-Ye Zhang nuclth: 1704.08503

21. Yubing Dong, Fei Huang, Pengnian Shen, Zongye Zhang, nucl-th:1704.01253

22. L.R. Dai, Y.N. Zhang, Y.L. Sun, S.J. Shao, Eur. Phys. J. A 52, 295 (2016).

23. C.S. An, H. Chen, Eur.Phys.J. A 52,2 (2016).

24. Fei Huang, Peng Nian Shen, Yu Bing Dong, Zong Ye Zhang, Sci. China Phys. Mech. Astron. 59, 622002 (2016).

25. M.N. Platonova, V.I. Kukulin, Phys.Rev. C 87, 025202 (2013).

26. P. Adlarson et al., Phys. Lett. B 774, 599 (2017)

27. Fan Wang, Jialun Ping, Hongxia Huang. arXiv:1711.01445

28. M. Bashkanov, H. Clement, T. Skorodko, Eur.Phys.J. A51 (2015) 7, 87.

29. A. Pricking, M. Bashkanov and H. Clement, arxiv:1310.5532 [nucl-ex].

30. M. Bashkanov, H. Clement, T. Skorodko, Nucl.Phys. A 958 129, (2017)

31. A. Fix, H. Arenhoevel, Eur. Phys. J. A 25, 115 (2005)

32. M. Egorov, A. Fix, Nucl. Phys. A 933, 104, (2015)

33. T. Ishikawa et al., Phys. Lett. B (2017), arXiv:1610.05532 .

34. M. Guenther, Master Thesis, University of Basel (2015).

35. M. Bashkanov et al., Phys. Lett. B 637, 223 (2006).

36. P. Adlarson et al., Phys. Rev. C 91, 015201 (2015).

37. P. Adlarson et al., Phys. Rev. C 86, 032201(R) (2012).

38. M. Bashkanov and H. Clement, Eur. Phys. J. A 50, 107 (2014).

39. I. Vidaña, M. Bashkanov , D.P. Watts, A. Pastore arXiv:1706.09701 
40. B.P. Abbott et al., Phys. Rev. Lett. 119, 161101 (2017).

41. S. J. Brodsky, C. R. Ji and G. P. Lepage, Phys. Rev. Lett. 51, 83 (1983); S. J. Brodsky and C. -R. Ji, Phys. Rev. D 33, 1406 (1986); Phys. Rev. D 34, 1460 (1986).

42. S. J. Brodsky and B. T. Chertok, Phys. Rev. Lett. 37, 269 (1976).

43. S. Rock, R. G. Arnold, P. E. Bosted, B. T. Chertok, B. A. Mecking, I. A. Schmidt, Z. M. Szalata and R. York et al., Phys. Rev. D 46, 24 (1992).

44. S. J. Brodsky, G. P. Lepage, Phys. Lett. B 87, 359 (1979); Phys. Rev. D 22 (1980) 2157.

45. A.V. Efremov, A.V. Radyushkin, Theor. Math. Phys. 42, 97 (1980); Phys. Lett. B 94 245, (1980).

46. Gerald A. Miller Phys. Rev. C 89, 045203 (2014) 